\title{
Data Mining Techniques Using Time Series Research
}

\author{
D. Senthil, G. Suseendran
}

\begin{abstract}
As time-series data are eventually large the discovery of knowledge from these massive data seems to be a challenge issue. The similarity measure plays a primary role in time series data mining, which improves the accuracy of data mining task. Time series data mining is used to mine all useful knowledge from the profile of data. Obviously, we have a potential to perform these works, but it leads to a vague crisis. This paper involves a survey regarding time series technique and its related issues like challenges, preprocessing methods, pattern mining and rule discovery using data mining. Streaming of data is one of the difficult tasks that should be managed over time. Thus, this paper can provide a basic and prominent knowledge about time series in data mining research field.
\end{abstract}

Keywords--- Data Streaming, Preprocessing Methods and Time Series Analysis.

\section{INTRODUCTION}

Data mining is used worldwide by many organizations effectively to improve their business processes, to provide better customer services, and to minimize costs. In case of predictive modeling, the input data must be regulated and maintained in the entity level. Such potential predictors are stored in a various format like time-stamped data tables as event based. For each event, a record is created based on the event in the table. So it seems to be a challenging issue for the data miners to transform the time-stamped data into predictive modeling. In this process time-stamped data is transformed into time series data there by creating information as potential predictors to exhibit predictive model ${ }^{1}$. This data preparation step is one of the primary tasks in time series analysis. One of the prominent areas of time series data mining is pattern detection. It exploits the identification of similarities in customer behavior, medical databases, agriculture, etc. For example, if an unusual behavior is seen in the time-based behavior of a particular customer, it automatically detects similar behavior in other customer data to reveal fraud activities. A well equipped data and extracted meaningful statistics discovers similar patterns among time series data or in transactional data to club those into several different groups. Such kinds of data can also be used as predictive model for weather forecasting tasks. In other words, data preparation, searching for similar time series, clustering and even forecasting within or beyond the segments are fundamental steps in time series data mining processes.

Manuscript received September 16, 2019.

D. Senthil, Ph.D, Research Scholar, Department of Computer Science, School of Computing Sciences, Vels Institute of Science, Technology \& Advanced Studies (VISTAS), Chennai. T.N, India. (e-mail: senthildphd@gmail.com)

Dr.G. Suseendran, Assistant Professor, Department of Information and Technology, School of Computing Sciences, Vels Institute of Science, Technology \& Advanced Studies (VISTAS), Chennai. T.N, India. (e-mail: suseendar_1234@yahoo.co.in)

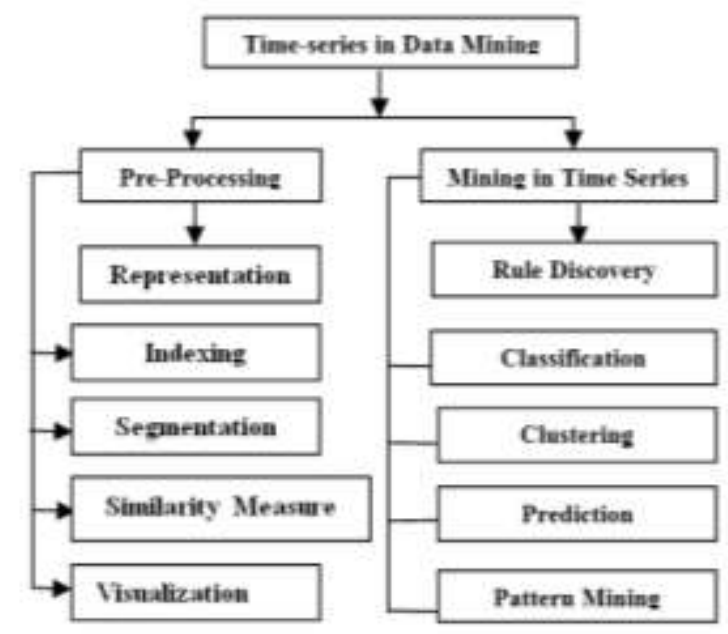

Figure 1: Overview of Time Series Analysis

Figure 1 illustrates the overview of time series analysis using data mining technique. It involves preprocessing and respective techniques to support it. Under preprocessing we have representation, indexing, segmentation, similarity measures and visualization technique. Rule Discovery, Classification, Clustering, Prediction and Pattern mining comes under mining of time series technique. A time series $\mathrm{T}$ is an ordered sequence of $\mathrm{n}$ real-valued variables $\mathrm{T}=$ $(t 1, \ldots \ldots, t n), € t i \in R$. Since time series is a kind of an underlying process in the course of which values are collected from measurements made at uniformly classified time instant for a given sampling rate. In other words, it can be defined as a set of contiguous time instants that can be univariate or multivariate when several series simultaneously span multiple dimensions within the same time range. Time series data covers the whole set of data generated through observation process. In the case of streaming, they are semi-infinite as time instants continuously feed the series. Thus, it is better to consider only the subsequences of a series ${ }^{2}$.

4,000 sample of graphics from world's 15 various newspapers published around 1974 to 1989 found that more than $75 \%$ graphics were made based on time series ${ }^{3}$ concept which is termed as a sequence of data points, measured at regular time intervals to specify the equidistant time units in terms of minutes, hours, days, and weeks. A time series time window defines the start value and end value where time dimension is sensitive to the predicting events. RFM (Recency, Frequency, and Monetary value), approach captures total support in several organizations based on customer marketing behavior. In data warehouse, the stored customer data does not provides prominent conclusion for predictive models. 
To modulate raw, time-stamped, and transactional data to the required data for predictive model, it is necessary to maintain a time interval suitable for the particular analysis in terms of hours, days and weeks at last aggregation of data is made for the required interval. It varies based on the application used. If the data miner, needs to verify all available aggregated statistics, which may lead to a substantial increase in the number of variables. Even though, several cross-sectional variables seen in a time series data, the analyst must decide the significant crosssectional variables suitable for aggregation using the data preparation method. Two time series methodology such as time series regression and structural time series are compared to model the number of road accidents in Penang. These two models involve different functionalities in terms of prominent factor that affects the number of road accidents. The structural time series model outperforms with better prediction rate ${ }^{4}$. Analysis of large amount of data can be done through various data mining methods based on their parameters and datasets according satisfy the user needs. These methods are classified as HDFS, Mapreduce and Hadoop environment with integration of $\mathrm{R}$ tool. Some Methods encompass sentimental analysis like NLP which increase the performance of density analysis. It accomplishes manual illustration and gradually advanced through storage and processing. Big data involves narrative techniques to manage information within limited runtime. To handle this circumstances unusual data is upgraded from the conventional data filtering techniques and adopt the new big data diagnostic tools ${ }^{5}$.

\section{PROBLEM INVOLVED IN TIME SERIES}

ime-arrangement databases is a succession database that include qualities or occasions that are gained through legitimate rehashed estimations of time, for example, securities exchange examination, monetary and deals anticipating, budgetary investigation, utility, stock, prepare, quality, yield and workload projections, control, perception of regular event like climate, temperature, wind, seismic tremor, different non specialized and specialized analyses, and restorative medications. Because of the development of an extensive number of sensors, telemetry gadgets, and other on-line information accumulation instruments, the measure of time-arrangement information expanding quickly as gigabytes every day and even every moment as that of NASA projects. To give connection inside timearrangement information and to discover designs, patterns, blasts and anomalies inside ideal time are is by all accounts a testing issue. In this segment, we look at a few parts of mining time-arrangement investigation, with an emphasis on pattern examination and likeness measures.

\section{PROPERTIES AND CHALLENGES OF LARGE TIME SERIES}

Before we see about different time arrangement information mining strategies, it is huge to comprehend what every one of the issues to be explained. Contingent upon a general lead, huge time arrangement join high dimensionality, clamor along trademark examples, anomalies and dynamism. Besides, the most key test in time arrangement information mining is the examination of at least two time arrangement moved or scaled as time or in plentifulness. The issues emerge from the properties of substantial time arrangement information. Firstly, if a perception of a period arrangement is seen as one measurement, the dimensionality of substantial time arrangement might be high 6 . This perception alone of time arrangement is by all accounts bigger than a few a great many perceptions can challenge. Working with super-high dimensional crude information can be extremely costly contrasted with handling and capacity costs7. Consequently, an abnormal state representation of the information or reflection is required. Regardless of, the fundamental reasoning of information mining indicates that shirking of potential data misfortune through the investigation of crude information is not fitting and moderate. In the regard of time arrangement information mining, clamor consolidated with trademark examples are terms to be repetitive sound 8 . With respect to the worldwide attributes, the time arrangement information mining methods should be powerful against uproarious segments. Huge measures of information are gathered to gauge mistakes of affectability towards and exceptions will be high. In the meantime, long time arrangement separates exceptions and uncommon results. In little subsamples anomalies are perceived as uncommon results and endeavors heterogeneity 9,10 .

As time arrangement information mining looks at least two time arrangement or subsequences it is not ceaselessly adjusted according to time hub or the sufficiency. In addition, fleeting and abundancy moving contrasts, time arrangement can have scaling or increasing speed contrasts with comparative attributes. Time arrangement information mining strategies must be vigorous against these changes and blends. Essentially, 1 trillion time arrangement objects require approximately 7:2 terabyte storage rooms. For delineation, a period arrangement with one trillion perceptions would compare to every single pulse of a 123 year old person. Another persuading explanation behind the use of time arrangement information mining strategies is the presence of such a huge measure of information that is too enormous to store. The approaching time arrangement information is becoming speedier than the capacity of people to process and store the crude information. Henceforth, the information must be diminished immediately to accomplish huge capacity size of the information as gushing information. For example, PC arrange track information or physical system sensors conveys non ceasing surges of data.

\section{Data Streaming}

Other than substantial time arrangement information sets the ceaseless stream information additionally brings more difficulties. Gushing information is portrayed by an enormous volume of incidentally requested perceptions shows up at a rapid rate, it is quick changing, and possibly unbounded. In such cases, if the whole unique information stream is too huge to store, the information mining strategies 
require various outputs of the information. Yet, continually rising information requires single-output and online multidimensional examination strategies for example and learning disclosure brings about the utilization of information decrease and ordering techniques. In any case, gushing information worried with information stream administration frameworks and considerably more huge and complex information sets are gathered step by step; the requirement for information digging systems for possibly unending volumes of spilling information is turning out to be extremely pressing. The trade between capacity size and precision for gushing information strategies makes much more critical than for time arrangement information mining when all is said in done. Spilling information strategies are still in the early phases of advancement and they need to achieve high pertinence in certifiable applications. Thusly, this exploration zone is one of the eminent points in time arrangement information mining.

\section{PREPROCESSING METHODS}

It is basic to preprocess the current information. As expansive time arrangement information is observed to be exceptionally massive and managing such information in its crude organization is costly as for preparing and capacity costs. Furthermore, we are managing time arrangement which are not clear in its crude configuration. Along these lines it is basic to lessen the dimensionality or section the time arrangement and after that file them. Appropriate representation methods and instruments must be used for expansive time arrangement crude information developed. Also, comparability measures are observed to be the foundation of all information mining applications.

\section{Indexing}

Representation and ordering procedures for time arrangement joined with each other. Time arrangement ordering plans are intended for effective time arrangement information association and particularly for a quick handling demand in expansive databases. To locate the nearest coordinate for a given question time arrangement $\mathrm{X}$ in a database, a successive or direct sweep of the entire database is exceptionally costly. Guide access to the crude information is wasteful as it can require a long investment. In this manner, ordering plans must be connected which is much quicker than consecutive or straight filtering of the time arrangement database. With a specific end goal to store two representation levels of the information, for example, crude information and a compacted abnormal state representation form of the information, it is important to execute a direct sweep for the question on the packed information and register a lower bound to the first separations to the inquiry time arrangement $\mathrm{X}$. as it were, the ordering is utilized to recover an outcome from the database. This brisk and clarity less outcome is utilized for a further examination of sub arrangements. Abstain from filtering the entire database however just look at specific groupings emerges issues. Building a record structure is much more productive likeness seek in a database. To gather comparable listed time arrangement into groups and get to just the most imperative bunches for further examinations. List structure methodologies can be further grouped into vector based and metric based record structures. Vector based list structures diminish the information dimensionality and structures bunch as vector based packed groupings. The bunching procedure can be even progressive and nonvarious leveled. R-Tree is the most prominent non-various leveled vector based file structure. For ordering, the initial few DFT coefficients, receive the $\mathrm{R} *$-tree. Later, 11 the exploration moved upon subsequence coordinating and utilize $\mathrm{R}^{*}$-trees as individually.

An outfit file joins at least two time arrangement representation methods for more compelling ordering. Taking after this Lastly, fractional occasional examples calculation in time arrangement databases has been introduced to address the issue of union mining A list structure in light of DFT coefficients take after empowers the wavelets based list structures. Consolidate mining strategy finds examples of at least two databases that are mined autonomously are blended. Clashing to this grouping in view of the packed components, metric based list structures bunch the arrangements in light of relative separations with each other. Multilevel separation based record structures are utilized for multivariate time arrangement where list suits numerous likeness measures and can be utilized for ordering multidimensional time arrangement. As said over, the decision of the list structure relies on upon earlier learning about the relating representation strategy.

\section{Segmentation}

The division issue can be settled in a few ways, consider the accompanying case, a period arrangement $\mathrm{T}$, deliver the best representation utilizing just $\mathrm{K}$ sections and furthermore it creates the greatest mistake for any fragment yet does not surpasses client indicated limit, for example, max-blunder. Despite the fact that $\mathrm{T}$, delivers the best representation to such an extent that the joined mistake of all portions is by all accounts not as much as client indicated limit, for example, add up to max-blunder. All calculations may bolster every one of these determinations. To produce a piecewise straight estimate, a calculation either freely rediscovered or some different methodologies recommended in related writing. Three noteworthy division approaches gives a broad experimental assessment on the heterogeneous accumulation of datasets like back, solution, assembling and science which brings about group calculation and delivers extremely poor approximations of the information that continually creates astounding outcomes and scales straightly as per the measure of information. This new online calculation that scales straightly in the measure of the information set, is observed to be on the web, and creates fantastic approximations is Sliding Window and Bottom-Up. Despite the fact that appearance of changed names and with marginally extraordinary execution points of interest, most time arrangement division calculations falls under three classes as demonstrated as follows. 
- Sliding Windows: A segment is grown until it exceeds the respective error bound. This process repeats with the next data point may not included in the newly approximated segment.

- Top-Down: The time series will be recursively partitioned until some stopping criteria have been reached.

- Bottom-Up: Starting from the finest possible approximation, segments are merged till the stopping criteria have been reached.

Approximation of time series with straight lines is classified as Linear Interpolation and Linear Regression to find the approximating line.

Linear Interpolation: The approximation line for the subsequence $\mathrm{T}[\mathrm{a}: \mathrm{b}]$ involves connection of $\mathrm{Ta}$ and $\mathrm{Tb}$ lines within constant time.

Linear Regression: The approximating line for the subsequence T[a: b] forms the best fitting line in the least

This can be acquired in time direct in the length of section results in disconnected look on some datasets. The stylish predominance of straight interjection, together with its low computational many-sided quality has settled on it the procedure of decision in PC realistic applications. In any case, the nature of the estimation line, as far as Euclidean separation, is regularly second rate compared to the relapse approach. All division calculations also require some technique to assess the nature of fit for a potential section. A measure usually utilized as a part of conjunction with direct relapse named as the whole of squares, or the remaining blunder. To accomplish this, consider the vertical contrasts between the best-fit line and the genuine information focuses, square them and afterward include it together. To quantify the integrity of fit, the separation between the bestfit line and the information point must be most extreme away in the vertical bearing (i.e. the L $\infty$ standard between the line and the information) notwithstanding time manysided quality. To begin with there is the topic of the correlation of significant division calculations. Whether the calculation is on the web or bunch. Also, there is the subject of how to determine the nature of required estimate. With inconsequential alterations the Bottom-Up calculation permits the client to determine the required estimation of $\mathrm{K}$, the most extreme mistake per portion, or aggregate blunder as guess. A non-recursive usage of Top-Down can likewise be made to bolster every one of the three choices. However Sliding Window just permits the greatest blunder according to fragment indicated ${ }^{12}$.

\section{Visualization} produced time arrangement for further examination by clients. It is likewise an intense apparatus to abuse the mining undertakings like example looking, inquiry byillustration, and example revelation a short time later. Current apparatuses for envisioning time arrangement are group and schedule based representation device, which presents lumps of information inside a given interim and afterward bunches them individually and winding perception instrument, maps the intermittent segment of time arrangement as a ring. These two devices are centered squares sense.

Perception is an imperative worldview to show the

around intermittent time arrangement and a settled length of period regarding week after week or month to month perspectives. Non-direct rescaling and space-effective rendering technique are even used to imagine the long time arrangement. Time Searcher is a period arrangement exploratory and perception instrument, used to recover time arrangement by questioning. In light of the current Time Boxes, which are rectangular, coordinate control time arrangement inquiries can be stretched out by presenting Variable Time Boxes, which permits the detail of questions in order to allow vulnerability in the time hub. Test occasions, Aggregated example occasions, Event record, and Interleave devent list are the four techniques that are to speak to the unevenly space time arrangement information. In addition, Time Searcher2 was created as another pursuit interface consolidating both channels and example look ability gave. Time Searcher is centered around numerous time arrangement question with the particular of concern district. Viz Tree is the sort of time arrangement representation apparatus that believers look numeric time arrangement to an image string in view of the SAX and an arrangement of sub strings (ie., gave a similar number of image) separated from the image string is encoded by an altered postfix tree along these lines pictures the recurrence of examples accessible. That is, the SAX discretizes the first run through arrangement into settled length subsequences, and change over pursuit subsequence to an image and the images acquired are included to frame an image string. Given an image string, say abccbccnnabcbccd, the following stride is to change over this long string to an arrangement of sub strings as per the length of every substring, W, determined by clients. For instance, if the favored length of substring is 4 , the given string will be separated into 4 substrings if bouncing window is utilized, i.e. abcc, bccn, nabc and bccd. Next, an addition tree will be built for the same. The length of substring mirrors the profundity of the tree where every branch of the tree speaks to an example. The recurrence of the example is spoken to by the thickness of every branch. Viz Tree produced in various applications indicates the subsequence coordinating, regularly showing up example disclosure and amazing example revelation. It is fit for finding every now and again showing up and amazing examples for a given determination, however suits just for time arrangement applications, for example, ECG. This same representation as in SAX gives distinctive perception apparatuses are likewise proposed by utilizing bitmap and speck plots. Multi-determination formats creates for long time arrangement in light of the idea of level of intrigue. In spite of, the work is further stretched out to find intriguing examples crosswise over various resolutions by receiving the typical representation of PIP rather than a SAX in the Viz Tree respectively ${ }^{13}$.

\section{MINING IN TIME SERIES \& RESULTS}

\section{Rule Discovery}

Run mining is another unmistakable assignment in the field of information mining. 
Affiliation lead mining is a standout amongst the most well known calculations. However the typical things have been introduced in the exchanges. Numerous scientists proposed new or adjusted calculations with regards to govern digging for time arrangement information. A minor approach includes discretizing the time arrangement information into fragments and changing over every section to an image. At that point principles can be found in the changed typical area group the subsequences to discover the images, and afterward basic control mining strategy has been connected to find the shrouded rules. Taking after this, a n-dimensional between exchange affiliation rules has being acquainted all together with handle both spatial and mixed media information mining. Fluffy affiliation guidelines are connected to the computational hypothesis of observation and flag handling strategies. A money related manage revelation strategy has been produced utilizing bullflag specialized diagramming heuristics 14 . With the effect of this, money related time arrangement is displayed in light of candle outlining strategy for run mining. At that point the exploration bunch confined a structure to find fleeting principles, which competent to create outofaset of successive examples in a state arrangement. It speaks to the fragments of time arrangement by characteristics and finds interim connections depicted regarding interim rationale. Control mining strategy in view of hereditary programming and concentrated equipment advances discretization develops time arrangement to foresee to rules. In spite of utilizing affiliation rules, choice tree is another straightforward approach for control mining. Preprocessing procedure finds fascinating standards from the medicinal time arrangement information. Aside from this, bunching system can likewise be embraced to find ordinary examples from subsequences for the discretization procedure. Since govern mining strategy depends on example extraction and choice tree the transient first rationale arrange frames changes consecutive crude information into successions of measures, then deduces fleeting standards utilizing the order trees for lead mining.

\section{Classification}

Time-arrangement information straightforwardly finds new closeness measures reasonable for it. At the point when contrasted with the grouping, the arrangement, the classes are known ahead of time and the calculation is prepared by the current dataset. Keeping in mind the end goal to realize, what are the special elements show up among them. At that point, when an unlabeled dataset is prepared utilizing the current framework to group to which class it has a place with. Change can be performed on the consecutive information to separate a component vector it must be sustained to the classifier to show the information. Different grouping calculations depend on giving new comparability measures. Also, the investigation of order calculations includes,

- Distance-based classification

- Feature-based classification

- Model-based classification

\section{Distance based classification}

Separate construct arrangement calculation is situated in light of, separation between information components case K-closest neighbor (KNN). Routine order calculations can be connected to time arrangement information with the assistance of the new closeness measures for consecutive information. Dynamic time distorting separation (DTW) is one of the Elastic closeness measures that are expected to comprehend this issue which shows mapping between nonstraight and two arrangement to minimize the separation between them. Despite the fact that numerous scientists acknowledged the predominance of DTW over Euclidean separation, since it's computational is wasteful and limits its reception. DTW is ascertained utilizing dynamic programming, thus has a quadratic time intricacy $\mathrm{O}(\mathrm{n} 2)$, as the explores planned to use this reality, as to the obliges, keeping in mind the end goal to accelerate the DTW counts. However, the more up to date calculations utilizes heuristic methodologies, which implies that however they are quicker in looking at arrangement, they don't yield appropriate ideal score record-breaking. Henceforth consecutive information can be multivariate. Separating of Multivariate Time Series (MTS) into discrete arrangement and preparing every one all alone creates connection between's those factors and advances a more up to date remove estimation calculation called Extended Frobenius standard, to oversee Multivariate Time Series. Besides, BLAST 2.0 instrument fuses BLAST motor to perform combine astute succession examination, later it is proposed as an option when looking at two arrangement that are now known to be homologous. Xing et al propose that separation measures indicate the precision of grouping calculation 15 highlight on its affectability to twisting in time. Bending is even non-straight, however direct change won't be adequate.

\section{Feature based Classification}

Highlight based arrangement calculations is a sort of grouping in light of list of capabilities, as ANN and Decision Tree calculations. Part Methods (KM) are likewise useful for highlight extraction, in the long run bargains image arrangement with different lengths. Where content information is considered as a pack of words instead of successive information, and underscored the capacity of portion strategies to manage printed information paying little respect to its immense number of components, ordinarily more than 10k. Since he was utilizing Support Vector Machine, which is one of the portion techniques however KM figures the inward result of the info vectors in a high dimensional space with respect to this, straight choice limits can be drawn between the classes. KM to perform arranges message as consecutive information. Like arrangement based separation measures, portion strategies are likewise generally utilized as a part of natural arrangement characterization. The decision of the appropriate components is the hardest piece of this procedure, to introduce the exchange off between these procedures physically by area specialists or include it as 
mechanized yet less exact as a rule. Examples and wavelet decay are a portion of the courses for separating highlights from successive information. Utilizing a discriminative approach, the parallel choices are considered as another arrangement has a place with a specific class or not, needed to utilize a choice trees for further order. More classes results in more branches and split focuses in the tree. Likewise, an example extraction calculation called Minimal Distinguishing Subsequence (MDS) is creates crevices inside the sub-arrangement, which makes it more reasonable to order utilizing natural arrangement. In any case another component extraction method is required to change the time-arrangement information into the recurrence area, so that the information dimensionality can be lessened further. For instance, consider Discrete Fourier Transform, DWT Discrete Wavelet Transform and Singular Value Decomposition strategies. Notwithstanding, Discrete Wavelet Transform is one of the basic in grouping that jam both time and recurrence attributes, though DFT gives just the recurrence qualities. Such change exceptionally well takes care of an issue, where it is important to know both nearby and expansive patterns inside the successive information. As DWT changes the information into various recurrence segments the higher request coefficients mirror the worldwide patterns of the information, while bring down request coefficients mirror the neighborhood patterns display in it. Recognizable proof of tree-leaves in view of their shapes is deluding by the misshapening in their shapes because of creepy crawlies eating parts of them. Rather than depending overall state of the leaves (worldwide elements); nearby components or examples decides the leaves from various trees. It is then changed over the shape information into a consecutive one. The principle point is to discover sub arrangement, or shapelets to separating between classes. To pick the subseries, all arrangement must be requested by their Euclidean separation from all conceivable shapelets. At that point it began to scan for a mid-point that partitions part arrangement of every class. With a specific end goal to apply highlight based arrangement to time arrangement information first it is expected to change consecutive information into list of capabilities.

\section{Model based Classification}

Show based techniques isolates the information into test and preparing information, and build a model utilizing preparing information and prepare the preparation dataset with the assistance of the model to order the preparation information assist. Models are being characterized into factual and neural system. Then again, the models are indicated as prescient models and enlightening models to anticipate inaccessible estimations of the information utilizing the current one and to discover examples and connections in the information, for instance Markov models were utilized a considerable measure in arrangement characterization applications Hidden Markov Model (HMM) is observed to be more effective in natural arrangement groupings, contrasted with Neural Networks, since it can manage variable-length arrangement, while the other procedure require settled length inputs however it additionally incorporate some of confinements. Well requires earlier space particular information to pick the

information highlights. By and large, Artificial Neural Networks are near factual models. As Recurrent Neural Networks (RNN) is considered as uncommon sort of ANN, where there is a criticism association in the system to monitor its inward state when managing new information sources. RNN is appropriate for successive information since, as per, RNN is fit for displaying the fleeting way of the grouping. Additionally, when contrasted with HMM, RNN does not require earlier information of the information and $\mathrm{RNN}$ is invulnerable to fleeting commotion. All things considered, as observed prior, it requires settled length inputs.

\section{Clustering}

Time arrangement grouping calculations frame bunches, in light of the sort of time arrangement information, qualifications depend on discrete-esteemed information or genuine esteemed information, consistently or nonconsistently examined one, univariate or multivariate, and regardless of whether the information arrangement are of equivalent length. Non-consistently examined information must be changed over into formally dressed information before grouping operations are performed. Different calculations have been produced for bunch distinctive sorts of time arrangement information. This study depicts the current time arrangement grouping techniques into three noteworthy classes, for example, crude information, by implication elements may separated from the crude information, or in a roundabout way models worked from the crude information.

\section{Raw-data-based clustering}

This classification includes crude information utilizing time or recurrence area. The two time arrangement tests are looked at a similar interim, yet their length regarding complete number of time focuses could possibly be same. To bunch the shifting multivariate information, it is important to alter the moved grouping method some time ago produced for static information. Progressive bunching and k-implies grouping strategies encourages the multivariate vector arrangement of seismic tremors and mining blasts. Non-stationary time arrangement are displayed with a period changing blend of stationary sources with the assistance of shrouded Markov demonstrate. Among determined number of bunches, as well as can be expected be discovered utilizing least summed up Ward foundation work. Notwithstanding this a separation work in light of the accepted free Gaussian models of information mistakes is created for a various leveled bunching technique keeping in mind the end goal to gathering regularity arrangement into an alluring number of groups. For the breaking down the element biomedical picture time arrangement information, deterministic tempering strategy is powerful for the insignificant free vitality vector quantization (VQ). Brief Time Series remove technique measures the comparability of the shape framed by the relative change of adequacy and the relating worldly data of uneven examining interims. 
Later bunching of non stationary time arrangement is finished by applying locally stationary renditions of Kullback-Leibler segregation data measures to give ideal time recurrence insights for measuring the error between two non-stationary time arrangement. Grouping multivariate time arrangement methodology performs two basic strides for both equivalent and unequal length. The initial step utilizes k-implies or fluffy c-implies grouping calculation to time stripped information and believers multivariate genuine esteemed time arrangement into univariate discreteesteemed time arrangement. The second step misuses kmeans or Fuzzy c-implies calculation to combine the changed over univariate time arrangement.

\section{Feature-based clustering}

This grouping incorporates rough data using time or repeat region. The two time course of action tests are taken a gander at a comparative between time, yet their length with respect to finish number of time centers could be same. To bundle the moving multivariate data, it is essential to modify the moved gathering technique some time prior delivered for static data. Dynamic packing and k-infers gathering techniques energizes the multivariate vector course of action of seismic tremors and mining impacts. Non-stationary time game plan are shown with a period changing mix of stationary sources with the help of covered Markov illustrate. Among decided number of packs, and in addition can be normal be found using minimum summed up Ward establishment work. Despite this a detachment work in light of the acknowledged free Gaussian models of data errors is made for a different leveled bundling strategy remembering the ultimate objective to social affair consistency course of action into an appealing number of gatherings. For the separating the component biomedical picture time plan data, deterministic treating procedure is capable for the immaterial free imperativeness vector quantization (VQ). Brief Time Series expel system measures the likeness of the shape encircled by the relative change of sufficiency and the relating common information of uneven inspecting between times.

Later grouping of non stationary time course of action is done by applying locally stationary versions of KullbackLeibler isolation information measures to give perfect time repeat bits of knowledge for measuring the mistake between two non-stationary time plan. Gathering multivariate time course of action system performs two essential steps for both proportional and unequal length. The underlying stride uses k-suggests or soft c-infers gathering computation to time stripped data and devotees multivariate authentic regarded time game plan into univariate discrete-regarded time course of action. The second step abuses k-means or Fuzzy c-infers count to join the changed over univariate time course of action.

\section{Model-based clustering}

In this sort of grouping, every time arrangement is produced by some sort of model or by a blend of fundamental likelihood circulations. Time arrangement are viewed as like describe the models if there should arise an occurrence of individual arrangement or the rest of the residuals in the wake of fitting the model. Particularly for the class of ARIMA invertible models grouping or browsing an arrangement of element structures can be accomplished through discovering Euclidean separation between their comparing autoregressive developments. Three metaheuristic strategies are assessed for dividing an arrangement of time arrangement into groups. To address the setting of melodic execution hypothesis, various leveled smoothing models called HISMOOTH models were acquainted with distinguish the relationship between the typical structure of a music score and its execution spoke to by a period arrangement. Taking after this, an agglomerative various leveled grouping technique in view of the p-estimation of a trial of speculation was connected to each combine of given stationary time arrangement. Bayesian calculation has being displayed to group the progression. Investigation of grouping of ARIMA time-arrangement, utilizing the Euclidean separation between the Linear Predictive Coding cepstra of two time arrangement abuses the uniqueness measure. Display based strategy likewise included for bunching univariate ARIMA arrangement. However the Gaussian blend model is utilized for speaker confirmation, a fluffy c implies grouping based standardization strategy is important to observe a superior score to be contrasted and a given limit along these lines tolerating or dismissing an asserted speaker ${ }^{16}$.

\section{Prediction}

Expectation or anticipating of few estimations of a period arrangement is a widely connected errand. A hypothesized causal relationship between factors are demonstrated and evaluated through information to estimate the following estimations of a period arrangement. Expectation of time arrangement qualities is an unmistakable and broad research territory separated from information mining, many surveys and standard references exist. Be that as it may, expectation is one of the significant patterns of information science. ARMA model is a standout amongst the most as often as possible utilized forecast systems and especially Seasonal Auto Regressive Integrated Moving Average models 17. To handle the issue required in long haul expectation and to join an immediate forecast techniques are coupled alongside very much shaped information determination criteria including $\mathrm{k}$-closest neighbor guess and nonparametric clamor estimation. Dynamic hereditary program was display particularly intended for guaging spilling information. Other than SARIMA models, much of the time utilized guaging methodologies are neural systems, Self Organizing Maps, or concealed Markov models. In addition, these expectation strategies are particularly framed for time arrangement information mining techniques. Creator Ahmed et al. presents outline of all machine learning approaches relevant to time arrangement anticipating, including multilayer perceptron, Bayesian neural systems, Radial Basis Functions, Kernel Regression, k-closest neighbor relapse, Regression Trees, Support Vector Regression Gaussian Process Regression, and so on., to demonstrate that Gaussian procedure relapses and multilayer perceptrons are 
the best strategies among them. Measurement decrease procedure accommodates multivariate time arrangement which concentrates on consistency. The Forecast able Component Analysis (ForeCA) approach lessens the measurement of multivariate time arrangement with the requirement for the most forecastable subspace. The audit of Goerg demonstrates that a lower entropy of the ghostly thickness infers a superior unsurprising sign and hence minimizes the entropy of the phantom thickness 18 . The self-sorting out Predictable Feature Analysis shaped on the comparative thoughts however extremely well looks for best unsurprising frameworks and not best unsurprising single parts as ForeCA method. In the mean time, the fluffy based techniques are connected for the forecast undertaking for non-stationary time arrangement. To finish this, firstly portion the time arrangement into subsequences utilizing Perceptually Important Points (PIP) and afterward look comparative subsequences utilizing Dynamic Time Warping (DTW). The mapping of the most comparable subsequence is likewise utilized for forecast. At last, an unsupervised learning calculation being proposed in view of hereditary programming for an occasion based expectation of time arrangement objects ${ }^{19}$.

\section{Pattern Mining}

As learning disclosure identifies the as often as possible showing up examples, curiosities and exceptions or freaks in the time arrangement of database. The oddity were resolved as oddities or by amazing examples. Design revelation is made with bunching strategies for event recurrence of the examples in time arrangement was accordingly found by the grouping. Themes are alluded as regular examples in a period arrangement database, they are observed to be subsequences of time arrangement which may like each other. In late research field, theme mining approach yields more noteworthy significance. The most famous example disclosure calculation includes Dynamic Time Warping method utilizing dynamic programming approach for this information revelation. Self Organizing Maps calculation is additionally utilized for the oddity location. As theme methodologies are require to predefined the theme length parameter and keep away from the failure shaped because of overwhelming commotion and poor adaptability in finding themes. Time arrangement datasets to intrinsic the intermittent structure, identifying periodicity to another sort of established example in the time arrangement examination strategies for taking care of regularity and periodicity. Time arrangement examination effectively handles gigantic datasets. Recognition of pattern conduct was subsumed to the general example is the discovery assignments. Set number of information in time arrangement looks into was expressly addresses recognizable proof of the continuous showing up patterns. The periodicity rate of time arrangement database and utilize control phantom thickness estimation is dug for the occasional example discovery. Additionally, the discovery of patterns is a traditional time arrangement investigation assignment also. Survey of Papadimitriou et al built up the mainstream Streaming Pattern revelation in numerous Time arrangement calculation to discover relationships and shrouded factors depicts the key patterns of the whole various time arrangement stream. Least Description Length guideline utilizes Principal Component Analysis for the extraction of themes from multi-dimensional time arrangement. In light of this, a theme disclosure calculation is created which is invariant to uniform scaling and first changes over the information to the SAX representation and after that attempt to discover anomalies20. Figure 2 determines the representation of example mining. The removed example showed up subsequent to preprocessing is nourished into the database to look at it. After examination handle the coordinated example is removed. A wavelet based tree structure has been presented for multi-level and level inquiries on time arrangement information and shocking examples are frequently alluded to as oddities. To accomplish this, an example is framed amazing just if the recurrence of its event contrasts altogether from the normal one. For building prospect, an addition tree is refined to encode the recurrence of every single watched example.

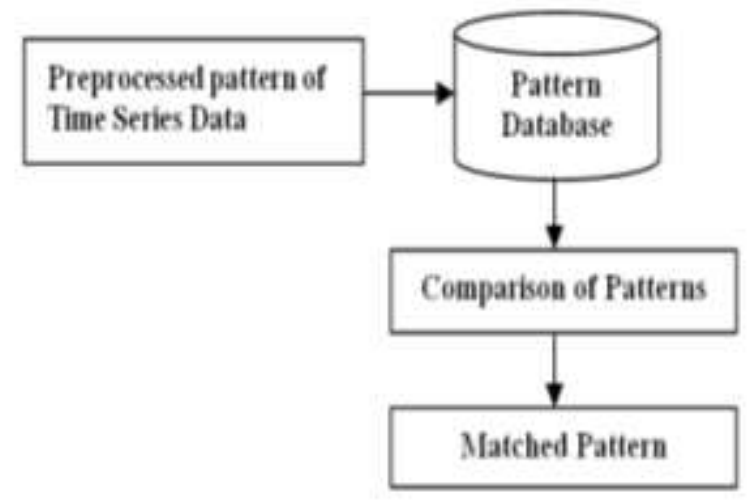

Figure 2: Pattern Mining

As the location of oddities ordinarily requires space situated mastery, a presumption free abnormality discovery approach is detailed in light of time arrangement bitmaps. Inconsistency scores are even created in an online way for numerous time arrangement. Uncommon time arrangement can be scaled through appropriate discovery of terabyte measured datasets with a circle mindful calculation. Late research field includes irregularity recognition and revelation of shocking examples in time arrangement. To gauge the money related working numbers it is expected to exchange monetary time arrangement information to fluffy examples and model them with fluffy semantic factors for example acknowledgment. Self-sorting out maps utilized as a part of securities exchange information for example disclosure. Peculiarities in time arrangement information are called as "frictions" indicated as subsequences that are not the same as every current subsequence. Besides theme approaches for time arrangement information mining prompts to unsatisfied interest for reasonable procedures however it productively handle multivariate time arrangement. Theme uses postfix trees to discover visit happening designs ${ }^{21}$. Molecule swarm is a computational enhancer that gives additionally astonishing examples have been seen as unmistakable from exception discovery. Exceptions encourage amazing information focuses and astonishing examples as accumulations of time arrangement

Published By: 
information focuses which are on the whole shocking or peculiarities. Perception of time arrangement themes infers variable lengths without earlier information about the themes. In the interim, creator accentuated on visual theme revelation since time arrangement conceals a colossal assortment of various incessant themes to bolster visual investigation. To take care of a similar issue, a parameter is proposed with the expectation of complimentary theme disclosure schedules. Creator Begum and Keogh detailed an oddity identification calculation in light of example densities and attempt to discover uncommon themes in time arrangement streams utilizing rough beast constrain calculation 22. As of late, a fluffy c-implies bunching calculation is connected to time arrangement information and isolated into subsequences with sliding windows to empower peculiarity location ${ }^{23}$.

\section{CONCLUSION}

The immense development of technology it is significant to store the maximum size and the complex datasets. The real-time time series of the datasets may involve the size up to the trillion observations and more. The goal is to extract new knowledge which is hidden in these large datasets. This survey provides the broad and deep knowledge regarding the challenges and techniques involved in time series data mining research field.

\section{REFERENCES}

1. Jiawei Han and Micheline Kamber, Data Mining, concepts and Techniques, second edition, Elsevier Publication, 2003.

2. Kumar Vasimalla. A Survey on Time Series Data Mining. International Journal of Innovative Research in Computer and Communication Engineering, 2014; 2(5):170-179.

3. Sascha Schubert, Taiyeong Lee. Time Series Data Mining with SAS Enterprise Miner. Data Mining and Text Analytics, SAS Global Forum, 2011: 1-12.

4. Noor Wahida Md Junus, Mohd Tahir Ismail, Zainudin Arsad. Predicting Penang Road Accidents Influences: Time Series Regression Versus Structural Time Series. Indian J Sci. Technol. 2016; 9(11): 1-18.

5. Amit Verma, Iqbaldeep Kaur, Namita Arora. Comparative Analysis of Information Extraction Techniques for Data Mining. Indian J Sci. and Technol. 2016; 9(11):1-18.

6. Rakthanmanon T, Campana B, Mueen A, Batista G, Westover B, Zhu Q, Zakaria J, Keogh E. Searching and mining trillions of time series subsequences under dynamic time warping. Proceedings of the 18th ACM SIGKDD international conference on Knowledge discovery and data mining, 2012: 262-270.

7. Fu TC. A review on time series data mining. Engineering Applications of Artificial Intelligence, 2011; 24(1): 164181 .

8. Esling $\mathrm{P}$, Agon C. Time-series data mining. ACM Computing Surveys (CSUR), 2012; 45(1):1-12.

9. Fan J, Han F, Liu H. Challenges of big data analysis. National Science Review, 2014: 293-314.

10. Caroline Kleist. Time Series Data Mining Methods A Review. Berlin, 2015.

11. Faloutsos C, Ranganathan M, Manolopoulos Y. Fast subsequence matching in time series databases. Proceedings of the1994 ACMSIGMOD International Conference on Management of Data, 1994: 419-429.
12. Tak-chung Fu. A review on time series data mining. Engineering Applications of Artificial Intelligence, 2011; 24:164-181.

13. Fu TC, Chung FL, Kwok KY. Stock time series visualization based on data point importance. Engineering Applications of Artificial Intelligence, 2008; 21(8): 1217-1232.

14. Leigh, W, Modani, N, Purvis, R, Roberts, T, Stock market trading rule discovery using technical charting heuristics. Expert Systems with Applications, 23, pp.155-159, 2002.

15. Xing Z, Pei J, Keogh E. A brief survey on sequence classification. ACM SIGKDD Explorations Newsletter. 2010; 12(1):40-48.

16. Warren Liao T. Clustering of time series data-a survey. Pattern Recognition, 2005; 38:1857 -1874.

17. Brockwell $\mathrm{P}$ J. Introduction to time series and forecasting. Taylor \& Francis. 2002; 1:1-12.

18. Goerg G. Forecastable component analysis. Proceedings of the 30th International Conference on Machine Learning (ICML-13), 2013; 64-72.

19. Kattan A, Fatima S, Arif M. Time-series event-based prediction: An unsupervised learning framework based on genetic programming. Information Sciences, 2015.

20. Yankov D, Keogh E, Medina J, Chiu B, Zordan V. Detecting time series motifs under uniform scaling. Proceedings of the 13th ACM SIGKDD international conference on Knowledge discovery and data mining, 2007: 844-853.

21. Floratou A, Tata S, Patel J M. Efficient and accurate discovery of patterns in sequence data sets. Knowledge and Data Engineering, IEEE Transactions, 2011; 23(8):1154-1168.

22. Begum N, Keogh E. Rare time series motif discovery from unbounded streams. Proceedings of the VLDB Endowment, 2014, 8(2).

23. Izakian H, Pedrycz W. Anomaly detection and characterization in spatial time series data: A clustercentric approach, 2014. 